\title{
Una definición operativa del desarrollo local para El Salvador
}

\author{
Diego Salcedo \\ Maestría en Desarrollo Local, \\ Departamento de Ciencias Politicas \\ UCA, San Salvador
}

RESUMEN: Los procesos de desarrollo a todos los niveles (locales, nacionales, regionales y globales) están influenciados por grandes corrientes de pensamiento, en términos de los valores adjudicados a las variables en juego. A la vez, podemos afirmar que no existe una unica teoria del desarrollo, sino distintas teorias que difieren entre ellas en la forma de considerar lo local. En este sentido, no se puede abordar el desarrollo local sin conocer de forma crítica las distintas teorias del desarrollo y los paradigmas que las fundamentan.

El desarrollo local en El Salvador tiene lugar a través de diversas iniciativas, tanto en el ámbito de los municipios como en el de las asociaciones, microregiones y mancomunidades de municipios, apoyadas por diversos actores locales, nacionales e internacionales. Estas iniciativas constituyen una riqueza de experiencias, que sin embargo no corresponden con los trabajos de sistematización, evaluación y retroalimentación de las mismas. Por lo tanto, existe un vacío de datos e información sistematizada, lo cual a la vez limita la capacidad de crear conocimientos sobre los enfoques y metodologías más adecuadas para apoyar el fin último de cualquier proceso de desarrollo local: aumentar la calidad de vida y el bienestar de la mayoría de la población humana que habita el territorio de la localidad. 
Con el fin de subsanar este vacío de conocimientos, un punto de partida es la necesidad de explicitar los supuestos teóricos que subyacen las distintas estrategias, políticas, programas y proyectos de desarrollo local en El Salvador. Además, existe la necesidad de comparar las distintas iniciativas en términos de sus resultados en cuanto a la mejora del bienestar y de la calidad de vida humana se refiere. Por lo tanto, se vuelve necesario medir el impacto de las mismas a través de indicadores que sean objetivamente verificables y comparables. Esto apunta a la necesidad de aplicar indicadores que midan la calidad de vida en el ámbito local (municipal y microregional), como por ejemplo el Índice de Desarrollo Humano (IDH) del Programa de las Naciones Unidas para el Desarrollo (PNUD).

Aunque parece existir un acuerdo o consenso básico sobre las múltiples dimensiones del desarrollo, que incluyen factores sociales, económicos, políticos, ecológicos y culturales, esto no implica que se sepa como gestionar dichos factores para lograr un mayor bienestar, incluyendo como principal factor limitante el de lograr un crecimiento económico que sea a la vez ambiental y socialmente sostenible.

Como se propone en el presente documento, esto implica la necesidad de adoptar un enfoque territorial que ayude a definir propuestas concretas de desarrollo a escala humana en el ámbito nacional, regional y local, que partiendo de los factores internos o endógenos, sepan aprovechar las oportunidades que ofrecen los fenómenos de la globalización y la localización ${ }^{1}$.

El objetivo de este trabajo de investigación es el de generar conocimientos sistemáticos en torno a los actores, conceptos y resultados del desarrollo local, tanto en términos del proceso como en términos de su impacto o contribución a la mejora de la calidad de vida. Por lo tanto, la necesidad de definir operativamente el desarrollo local en El Salvador se plantea como un intento de contribuir conceptualmente a la valoración de los distintos procesos de desarrollo local que se encuentran ubicados en nuestro territorio nacional (políticas, estrategias, programas y proyectos, etc.).

\section{Orígenes del concepto del desarrollo}

En la época moderna, marcada por el surgimiento y consolidación de los Estado nación en la Europa occidental de los siglos XIII al 
XIX, el concepto del desarrollo aparece por primera vez como política estatal por su vinculo con los intereses militares de conquista o defensa de dichos Estados. Según Rostow (1975): "Los gobernantes europeos percibían...que sus intereses en el ingreso impositivo para fines militares requerían de una expansión de la producción."2 Por lo tanto, la ampliación de la riqueza para fines militares, a través del incremento constante de la producción, se vuelven bienes legítimos de la política del Estado. Sin embargo, es en el discurso de la toma de posesión de 1949 del presidente de los Estados Unidos de América (EEUU), Harry Truman, que nace el concepto contemporáneo del desarrollo, entendido como la aplicación de los avances científicos y tecnológicos logrados por EEUU en el campo de la industria en el resto del mundo, como única medida del progreso:

"Debemos emprender un nuevo programa audaz que permita que los beneficios de nuestros avances científicos y nuestro progreso industrial sirvan para la mejoría y el crecimiento de las áreas subdesarrolladas... El viejo imperialismo - la explotación para beneficio extranjero- no tiene ya cabida en nuestros planes. Lo que pensamos es un programa de desarrollo basado en los conceptos de un trato justo democrático."3

Así, después de la recién finalizada segunda guerra mundial, el mundo se dividía en el primer mundo de los países ricos e industrializados, liderado por EEUU y materializado a través del Plan Marshall en la reconstrucción europea, y en el mundo subdesarrollado de los países pobres, o tercer mundo. En medio quedaba el denominado socialismo real de la URSS, quienes a pesar de su confrontación ideológica con el sistema capitalista y la sociedad de mercado de EEUU, seguían los mismos patrones de industrialización del mundo capitalista occidental.

Según Arndt (1992), lo nuevo no era el desarrollo económico como proceso histórico, sino la aceptación de que los países que hasta ese momento habían sido relegados, deberían tratar de participar en él y recibir ayuda al hacerlo. ${ }^{4}$ En este sentido, se puede afirmar que las teorías contemporáneas referidas al desarrollo surgen hacia 1945, a finales de la segunda guerra mundial, ${ }^{5}$ aunque según Rubio (1991), dichas teorías, generadas y aplicadas aproximadamente entre 1945 y 1965, no constituyen verdaderas teorías del desarrollo, sino más bien 
deberían considerarse simplemente como teorías de crecimiento económico, ya que en las mismas los países subdesarrollados no aparecen como objeto de estudio. " Se les considera que son países "en retraso", sin historia propia, que simplemente deben seguir el camino trazado por los países "desarrollados". Por lo tanto, durante dicho periodo (1945-1965), el término desarrollo se consideraba simplemente como un sinónimo de crecimiento económico.

Según Blomstrom y Hettne (1984), el desarrollo económico del tercer mundo nace como objetivo de guerra y posguerra de los gobiernos de los países aliados occidentales, como una forma de ganarse la opinión pública y de motivar el optimismo de sus propios países a través del idealismo de planes para un mundo mejor de posguerra.?

Según Myrdal (1957): "La aparición en los países subdesarrollados de este impulso común hacia el desarrollo económico como fin político superior, la definición del desarrollo económico como la elevación de los niveles de vida de la gente común, el acuerdo de que el desarrollo económico es una tarea para el gobierno...representa algo totalmente nuevo en la historia." ${ }^{8}$ En este sentido, se considera que las naciones desarrolladas son las naciones industrializadas, las cuales deben ser emuladas por el resto de países en su evolución hacia el desarrollo.

Por lo tanto, dicho enfoque parte de una visión euro céntrica y lineal del progreso, donde existe el conocimiento del punto de llega$\mathrm{da}$, entendido como la industrialización o modernización, así como de las etapas a seguir, resumidas por su máximo exponente, Rostow (1960), de la siguiente manera: ${ }^{9}$

1. Sociedad tradicional (bajo nivel tecnológico)

2. Etapa anterior al despegue (modernización)

3. Despegue (ahorro interno e industrialización)

4. Camino a la madurez (reindustrialización)

5. Sociedad de consumo masivo (satisfacción plena de las necesidades básicas; producción orientada hacia los bienes durables y servicios)

Según Rostow, todas las sociedades del planeta pasarían por dichas etapas, lo cual les permitiría alcanzar el desarrollo. Según este enfoque de evolución lineal y universal, las sociedades locales pueden en- 
trar en la marcha hacia el desarrollo si se adaptan a las exigencias del modelo industrial, es decir, a la introducción de las tecnologías industriales que han demostrado su eficacia en otras latitudes, aunque esto signifique suprimir características locales importantes.

Sin embargo, los años sesenta también marcan el fin del optimismo desarrollista, dando paso a las crisis económicas acentuadas por la crisis internacional del petróleo, resultantes en la sensible reducción de las tasas de crecimiento económico experimentadas hasta entonces, y poniendo en duda la premisa del crecimiento económico ilimitado. De igual forma, la creciente polarización en el ámbito global se da ya no solo en el contexto de la guerra fría (Capitalismo, ie. EEUU y sus aliados en Europa occidental vs. Comunismo, ie. URRS y sus aliados), sino también en el contexto de las naciones ricas contra las pobres, o de Norte vs. Sur.

Adicionalmente, se dan una serie de críticas académicas al paradigma del desarrollo como crecimiento económico ilimitado, provenientes tanto de adentro de los países del norte o centro (los movimientos ecologistas), como desde los países del sur, o periferia, y en particular de América Latina (ej. CEPAL). Según Rubio (1991), esto hace que las teorías del crecimiento económico den paso a las teorías del desarrollo entendidas como "occidentalización, modernización e industrialización", y también a que se abandone la idea de formular una única teoría del desarrollo, existiendo diferentes enfoques. ${ }^{10}$

Según Esteva (1992):"1 "A finales de la década [de los 60], muchos factores contribuyeron a enfriar el optimismo sobre el crecimiento económico: se hicieron más perceptibles que al principio de la década las deficiencias de las políticas y los procesos en curso; se ampliaron los atributos que debían ser integrados; y se hizo claro que el crecimiento rápido estaba siempre acompañado de crecientes desigualdades. Para entonces, los economistas se sentían más inclinados a reconocer los aspectos sociales como 'obstáculos sociales.' Evidencias uniformes permeaban a los órganos oficiales:

El hecho de que el desarrollo deje atrás, o incluso cree de alguna manera, grandes zonas de pobreza, estancamiento, marginalidad y exclusión real del progreso económico y social es demasiado obvio y urgente para dejarse de lado. ${ }^{12}$ 
Cinco años antes de que Truman iniciara la era del desarrollo, Karl Polanyi escribía sobre los orígenes y consecuencias del sistema de industrialización capitalista, a partir de la imposición de los principios de la economía de mercado como guía para la conducción de la sociedad. ${ }^{13}$

Esta "gran transformación", como la llama Polanyi, significaba algo totalmente nuevo en la historia de la humanidad, dado que hasta entonces la economía siempre había estado imbricada y supeditada a las instituciones sociales, y no viceversa. Sin embargo, con la apropiación de las tierras comunales, la revolución industrial, y la sociedad de mercado surgida del sistema mercantilista en Inglaterra entre los siglos XVII y XIX, y luego difundido por el mundo entero por el imperio británico, la sociedad se concibe en función de las necesidades de la economía de mercado.

Según Arndt: "Desde [Gran Bretaña] la modernización se extendió, primero, a otras naciones de Europa Occidental y, por su intermedio, a las colonias europeas de ultramar y luego, antes de que finalizara el siglo XIX, hasta Rusia y Japón." ${ }^{14}$ Según List (1845), el auge y la expansión del imperio británico se debió a una serie de medidas de política aplicadas por sus gobernantes para desarrollar el vínculo entre su agricultura e industria, incluyendo medidas para la atracción de los recursos humanos más capacitados provenientes de otras naciones, así como medidas de apoyo económico, es decir, subsidios, y de protección interna o aranceles para sus bienes manufacturados, a manera de asegurarles la cautividad de su mercado interno. ${ }^{15}$

List argumenta y demuestra con los datos históricos disponibles en su época (hace más de 150 años) que el libre comercio no es siempre beneficioso, tal y como asumía erróneamente Adam Smith, sino que depende del nivel de desarrollo comparativo entre las naciones, saliendo más beneficiada con el libre intercambio la nación más avanzada. Para List, el gran error de Smith consistía en que su teoría ignoraba la existencia de distintas naciones con distintos niveles de desarrollo e intereses contrapuestos. Así, List demuestra como la teoría del libre intercambio de Smith funcionaría solo en un mundo ideal en el que todas las naciones tuvieran el mismo nivel comparativo de desarrollo de su agricultura, industria y comercio mercantil, lo cual por supuesto no coincide con la realidad histórica. 
Adicionalmente, List demuestra como el imperio británico se dedicó activamente a promover el comercio internacional de sus bienes manufacturados a cambio de materias primas provenientes ya sea de sus colonias o de otras naciones, incluyendo el proteccionismo comercial y el dominio de las rutas mercantes por medios militares y por su apoyo a la piratería, en función de los intereses británicos. Inglaterra incluso llegó a prohibir el desarrollo de la industria manufacturera en sus colonias, para asegurarle también este mercado cautivo a su industria nacional. Al mismo tiempo que actuaban de forma intervencionista y proteccionista, apegados internamente a su propia Acta de navegación, el imperio británico se dedicaba a promulgar las bondades de la teoría del libre comercio elaborada por Adam Smith, utilizándola como un poderoso argumento para abrir los mercados de las otras naciones de Europa, a través de tratados de libre comercio.

Por su parte, Polayni nos demuestra que el determinismo económico es un fenómeno del siglo XIX, que el sistema de mercado distorsionó violentamente nuestras concepciones del hombre y la sociedad, y que estas concepciones distorsionadas resultaban ser los principales obstáculos para la solución de los problemas de nuestra civilización. ${ }^{16}$ En este sentido, tanto la tierra como los mismos seres humanos, llegan a ser considerados como mercancías.

Polanyi documentó cuidadosamente la historia económica de Europa como la historia de la creación de la economía como una esfera autónoma, desligada del resto de la sociedad. Mostró que el mercado nacional no apareció como la emancipación gradual y espontánea de la esfera económica del control gubernamental, sino precisamente lo contrario: el mercado fue el resultado de una intervención consciente y a menudo violenta del gobierno. ${ }^{17}$ Hace mas de 50 años, Polanyi hacía esta sobria declaración: "Permitir que el mecanismo del mercado gobierne en exclusiva el destino de la humanidad y su ecosistema natural... conllevaría la devastación de la sociedad."18

No obstante, Polanyi se mostraba convencido de que tal desastre era impensable en el mundo en el periodo de la postguerra porque, según decía: "Somos testigos de que se está dando un tipo de desarrollo entre las naciones en el que el sistema económico deja de dictar las leyes a la sociedad, luego, la hegemonía de la sociedad sobre el sistema esta garantizada. ${ }^{19}$

57

Una definición operativa del desarrollo local para El Salvador 
Polanyi decía esto a raíz de lo que el pensaba que los dirigentes políticos del mundo habían aprendido después de la guerra, a partir de la experiencia de la recién finalizada época liberal, la cual había demostrado que la sociedad, al ser supeditada a la economía de mercado, había llevado a la crisis económica más severa de la historia, la gran depresión, y había desembocado en dos guerras mundiales.

Por lo tanto, la correlación de las fuerzas políticas de entonces (compuesta por una combinación de democristianos, socialdemócratas, keynesianos y marxistas), favorecían una fuerte intervención del Estado para controlar las fuerzas del mercado, en función de los intereses de la sociedad, y apuntaban a que el liberalismo no era una opción política viable.

\section{Nuevas aproximaciones al concepto del desarrollo}

Según Esteva (1992): “Conceptualmente, existía una rebelión generalizada contra la camisa de fuerza de las definiciones económicas del desarrollo, que constreñían sus metas a indicadores cuantitativos más o menos irrelevantes. El presidente del Banco Mundial, Robert S. McNamara, planteó muy claramente el asunto en 1970. Tras reconocer que una alta tasa de crecimiento no había traído consigo un progreso satisfactorio en el desarrollo durante la Primera Década del Desarrollo de Naciones Unidas (1960-1970), insistió en que la de 1970 debía contener algo mas que medidas burdas de crecimiento econó$\operatorname{mico}^{20} \ldots$

La búsqueda del principio unificador se desplazó a otro terreno. En 1974 la Declaración de Cocoyoc puso el acento en que el propósito del desarrollo 'no debe ser desarrollar las cosas, sino al hombre.' 'Cualquier proceso de crecimiento,' añadió, 'que no conduzca a la satisfacción (de las necesidades básicas) - o que, aun peor, la perturbe- es una parodia de la idea de desarrollo.' La Declaración también subrayó la necesidad de la diversidad y de 'seguir muy diferentes caminos al desarrollo,' así como la meta de la autosuficiencia y el requerimiento de 'cambios económicos, sociales y políticos fundamentales. ${ }^{21}$ Algunas de estas ideas se ampliaron después en las propuestas de la Fundación Dag Hammarskjold, la cual planteó, en 1975, otro desarrollo. ${ }^{22}$ 
En 1976, la satisfacción de las necesidades básicas de la población de cada país definió la parte central y primera del Programa de Acción de la Conferencia Mundial Tripartita sobre Empleo, Distribución del Ingreso y Progreso Social de la OIT. Los expertos de UNESCO, por su parte, promovieron el concepto de desarrollo endógeno. Por algún tiempo, esta concepción ganó aceptación sobre las demás. Parecía claramente herética, en abierta contradicción con la sabiduría convencional. A partir de una crítica rigurosa de la hipótesis del desarrollo 'en etapas' (Rostow), la tesis del desarrollo endógeno rechazó la necesidad o la posibilidad — por no hablar de la conveniencia- de imitar mecánicamente a las sociedades industriales. Propuso, en vez de ello, tomar debidamente en cuenta las particularidades de cada nación. ${ }^{23 "}$

\subsection{El desarrollo humano sostenible}

Según el Programa de las Naciones Unidas para el Desarrollo en El Salvador (PNUD, 2003), el punto de partida del "enfoque del desarrollo humano" es que "la verdadera riqueza de una nación está en su gente." ${ }^{24}$ El objetivo clave del desarrollo es la ampliación de la participación, la cual solo es posible por la expansión de las oportunidades y de las capacidades. Las personas, por lo tanto, pasan a ser consideradas como el foco central de la gestión y constituyen el fin hacia el cual deben ir dirigidos todos los análisis y políticas. Este enfoque usa indicadores de carácter multidimensional para medir las metas y logros, tales como el Índice de Desarrollo Humano (IDH), el Índice de Pobreza Humana (IPH), el Índice de Desarrollo relativo al Género (IDG) y el Índice de Potenciación de Género (IPG).

El lanzamiento del primer Informe sobre Desarrollo Humano en 1990 se produjo en un contexto histórico caracterizado por tres procesos simultáneos que lo revisten de importancia.

Primero, "la consolidación del predominio de las concepciones ideológicas y estrategias basadas en el libre mercado para lograr el crecimiento económico, el cual se identificaba como sinónimo del desarrollo, por sobre cualquiera de las otras posibles estrategias de gestión de los asuntos públicos."25

Segundo, "la globalización, que en la creciente interconexión e interdependencia de la humanidad a través de mayores flujos de inver-

Una definición operativa del desarrollo local para El Salvador 
sión y comercio, expresaba el surgimiento de nuevos mercados de divisas y de capitales, nuevos instrumentos de comunicación (i.e. Internet, teléfonos celulares, redes multimedia), nuevos actores (i.e. Empresas transnacionales, ONG internacionales, la Organización Mundial del Comercio, OMC) y nuevos marcos normativos más obligatorios para los gobiernos nacionales (i.e. Acta Final de la Ronda de Uruguay/OMC, Tratados de Libre Comercio-TLC)." ${ }^{26}$

Tercero, "la finalización de la guerra fría, que mantuvo dividida a la humanidad entre dos visiones que competían por el futuro del mundo: la del mundo capitalista, que cuestionaba a los países socialistas por denegar los derechos civiles y políticos; y a del mundo socialista, que hacía énfasis en la primacía de los derechos económicos y sociales, y criticaba a los países capitalistas de generar grandes desigualdades y exclusiones."27

La globalización y el fin del socialismo crearon un entorno favorable para colocar en una posición de predominio a la propuesta liberal para el desarrollo, sintetizada en el denominado "Consenso de Washington."

Dicha propuesta incluye un conjunto de planteamientos con relación a los objetivos, estrategias, prioridades y políticas de desarrollo. El objetivo a perseguir es maximizar el bienestar económico, para lo cual se considera indispensable perfeccionar el funcionamiento de los mercados y lograr así mayores niveles de eficiencia económica y de crecimiento del PIB. Reconoce que los derechos humanos y la vigencia de un Estado democrático son elementos claves para la gobernabilidad, pero aboga por un Estado reducido a su mínima expresión. En cuanto a prioridades del desarrollo, sus énfasis están puestos en el crecimiento económico y en las inversiones - consideradas de alta rentabilidad - en educación, salud y redes de seguridad social. Finalmente, sus propuestas se concentran en un decálogo de políticas, con especial énfasis en la liberalización comercial, la desregulación y las privatizaciones.

Desde el enfoque del desarrollo humano, se hacen actualmente dos críticas principales a las políticas promovidas por el Consenso de Washington. Primero, que han fracasado en generar el crecimiento económico que los países necesitan para reducir la pobreza y enfrentar otros desafíos propios del desarrollo. Segundo, que donde se ha 
dado algún crecimiento económico, éste se ha dado a costa de aumentos en la pobreza, agudización de la desigualdad, migraciones masivas y mayor degradación ambiental.

Otra contribución del enfoque de desarrollo humano es que la calidad del crecimiento económico es tan importante como su cantidad. El concepto de desarrollo humano plantea que no existe un vínculo automático entre el aumento del ingreso y la ampliación de las opciones humanas. Aunque el ingreso crezca, si éste no es distribuido equitativamente, habrá grupos para los cuales las opciones económicas seguirán siendo iguales o aún más limitadas. Algo similar podría suceder si dentro del presupuesto de los gobiernos no se reconoce debidamente la prioridad que debe tener el acceso universal a los servicios sociales básicos (agua potable, educación, y salud).

El éxito que hasta ahora ha tenido el paradigma del desarrollo humano se debe fundamentalmente a dos cosas: 1) Haber introducido un indicador del desarrollo del mismo nivel de simplicidad que el Producto Interno Bruto (PIB) per cápita (un solo número), que no es ciego a los aspectos sociales de las vidas humanas; 2) Haber proporcionado oportunamente el enfoque multidisciplinario de desarrollo humano como una alternativa al enfoque economicista promovido por los partidarios del mercado libre.

Entre las críticas que se le hacen al enfoque del desarrollo humano del PNUD, Esteva afirma que "el informe sigue claramente los pasos de quienes intentan cuantificar lo económico, aunque presta apropiada consideración a los esfuerzos de Unrisid por medir y analizar el desarrollo socioeconómico y la tradición de los Informes sobre la situación social en el mundo" 28 , refiriéndose en particular al primer informe de 1990. Adicionalmente, por su énfasis en la medición del desarrollo como nivel de logro, el enfoque del desarrollo humano del PNUD se critica por su incapacidad de tomar en cuenta los factores intangibles del desarrollo, tales como las capacidades humanas, así como la inter-subjetividad del concepto del desarrollo.

\subsection{Desarrollo a escala humana: necesidades y satisfactores}

Uno de los mitos creados por la sociedad económica en la que vivimos consiste en creer que nuestro bienestar aumenta en la medida en que consumimos más bienes y servicios, dado que los mismos 
sirven para satisfacer la infinidad de necesidades humanas. En tal sentido, entre más bienes y servicios consumimos, más felices somos, dado que así logramos satisfacer más de nuestras necesidades. Una primera falacia es la de suponer que las necesidades humanas son infinitas. La segunda falacia consiste en creer que dichas necesidades se satisfacen únicamente a través de bienes y servicios económicos provistos por el mercado. La tercera falacia consiste en obviar que la satisfacción de ciertas necesidades por parte de unos restringe la capacidad de otros de satisfacer las propias, e incluso la satisfacción de otras necesidades propias.

Manfred Max-Neef nos propone una forma de superar este mito. Según nos explica en su libro Desarrollo a escala bumana: "se ha creído, tradicionalmente, que las necesidades humanas tienden a ser infinitas; que cambian constantemente, que varían de una cultura a otra y que son diferentes en cada período histórico. Nos parece que tales suposiciones son incorrectas, ya que son producto de un error conceptual. El típico error que se comete en los análisis acerca de las necesidades humanas es que no se explica la diferencia esencial entre las que son propiamente necesidades y los satisfactores de esas necesidades. Es indispensable hacer una distinción entre ambos conceptos por motivos tanto epistemológicos como metodológicos. La persona es un ser de necesidades múltiples e interdependientes. Las necesidades humanas pueden dividirse conforme a múltiples criterios, y las ciencias humanas ofrecen en este sentido una vasta y variada literatura." ${ }^{29}$

Max-Neef combina dos criterios posibles de división: según categorías existenciales y según categorías axiológicas. Esta combinación permite reconocer, por una parte, las necesidades de ser, tener, hacer y estar; y, por la otra, las necesidades de subsistencia, protección, afecto, entendimiento, participación, ocio, creación, identidad y libertad. Ambas categorías de necesidades pueden combinarse con la ayuda de una matriz (ver Cuadro 1).

Según Max-Neef, no existe una correspondencia biunívoca entre necesidades y satisfactores. Un satisfactor puede contribuir simultáneamente a la satisfacción de diversas necesidades; a la inversa, una necesidad puede requerir de diversos satisfactores para ser satisfecha... La matriz que refleja el cuadro 1 no agota los tipos de satisfactores posibles. De hecho, estos abarcan un gran abanico de 


\section{Cuadro 1}

\begin{tabular}{|c|c|c|c|c|}
\hline $\begin{array}{l}\text { Necesidades } \\
\text { según categorias } \\
\text { existenciales }\end{array}$ & SER & TENER & HACER & ESTAR \\
\hline \multicolumn{5}{|l|}{$\begin{array}{l}\text { Necesidades } \\
\text { según categorias } \\
\text { axiológicas }\end{array}$} \\
\hline SUBSISTENCIA & $\begin{array}{l}\text { Salud física, } \\
\text { salud mental, } \\
\text { equilibrio }\end{array}$ & $\begin{array}{l}\text { Alimentación, } \\
\text { abrigo, trabajo }\end{array}$ & $\begin{array}{l}\text { Alimentar, } \\
\text { trabajar, } \\
\text { descansar }\end{array}$ & Entorno vital \\
\hline PROTECCION & $\begin{array}{l}\text { Cuidado, } \\
\text { adaptabilidad, } \\
\text { auto nomia, } \\
\text { equilibrio, } \\
\text { solidaridad }\end{array}$ & $\begin{array}{l}\text { Sistemas de } \\
\text { seguro, ahorro, } \\
\text { seguridad } \\
\text { social, sistemas } \\
\text { de salud, } \\
\text { legislaciones, } \\
\text { derechos, } \\
\text { familia, trabajo }\end{array}$ & $\begin{array}{l}\text { Cooperar, } \\
\text { prevenir, } \\
\text { planificar, } \\
\text { cuidar, curar, } \\
\text { detener }\end{array}$ & $\begin{array}{l}\text { Contorno vital, } \\
\text { contorno social, } \\
\text { morada }\end{array}$ \\
\hline AFECTO & $\begin{array}{l}\text { Autoestima, } \\
\text { solidaridad, } \\
\text { respecto, } \\
\text { tolerancia, } \\
\text { generosidad, } \\
\text { receptividad, } \\
\text { pasión, } \\
\text { voluntad, } \\
\text { sensualidad, } \\
\text { humor }\end{array}$ & $\begin{array}{l}\text { Amistades, } \\
\text { pa rejas, familia, } \\
\text { animales, } \\
\text { plantas, } \\
\text { jardines }\end{array}$ & $\begin{array}{l}\text { Hacer el amor, } \\
\text { acariciar, } \\
\text { expresar } \\
\text { emociones, } \\
\text { compartir, } \\
\text { cuidar, cultivar, } \\
\text { apreciar }\end{array}$ & $\begin{array}{l}\text { Privacidad, } \\
\text { intimidad, } \\
\text { hogar, espacios } \\
\text { de encuentro }\end{array}$ \\
\hline ENTENDIMIENTO & $\begin{array}{l}\text { Conciencia } \\
\text { crítica, } \\
\text { receptividad, } \\
\text { curiosidad, } \\
\text { asombro, } \\
\text { disciplina, } \\
\text { intuición, } \\
\text { racionalidad } \\
\end{array}$ & $\begin{array}{l}\text { Literatura, } \\
\text { maestros, } \\
\text { método, } \\
\text { políticas } \\
\text { educacionales, } \\
\text { políticas } \\
\text { comunicaciona } \\
\text { les }\end{array}$ & $\begin{array}{l}\text { Investigar, } \\
\text { estudiar, } \\
\text { experiment ar, } \\
\text { educar, } \\
\text { analizar, } \\
\text { meditar, } \\
\text { interpretar }\end{array}$ & $\begin{array}{l}\text { Ambitos de } \\
\text { interacción } \\
\text { formativa: } \\
\text { escuelas, } \\
\text { universidádes, } \\
\text { academias, } \\
\text { agrupaciones, } \\
\text { comunidades, } \\
\text { familia } \\
\end{array}$ \\
\hline PARTICIPACIÓN & $\begin{array}{l}\text { Adaptabilidad, } \\
\text { receptividad, } \\
\text { solidaridad, } \\
\text { disposición, } \\
\text { convicción, } \\
\text { entrega, } \\
\text { respeto, } \\
\text { pasión, humor }\end{array}$ & $\begin{array}{l}\text { Derechos, } \\
\text { responsabilida- } \\
\text { des, } \\
\text { obligaciones, } \\
\text { atribuciones, } \\
\text { trabajo }\end{array}$ & $\begin{array}{l}\text { Afiliarse, } \\
\text { cooperar, } \\
\text { proponer, } \\
\text { compartir, } \\
\text { discrepar, } \\
\text { aclarar, } \\
\text { dialogar, } \\
\text { acordar, opinar }\end{array}$ & $\begin{array}{l}\text { Ámbitos de } \\
\text { interacción } \\
\text { pa rticipativa, } \\
\text { cooperativa, } \\
\text { asociaciones, } \\
\text { iglesias, } \\
\text { comunidades, } \\
\text { vecindarios, } \\
\text { familia } \\
\end{array}$ \\
\hline OCIO & $\begin{array}{l}\text { Curiosidad, } \\
\text { receptividad, } \\
\text { imaginación, } \\
\text { despreocupación, } \\
\text { humor, } \\
\text { tranquilidad, } \\
\text { sensualidad }\end{array}$ & $\begin{array}{l}\text { Juegos, } \\
\text { espectáculos, } \\
\text { fiestas, calma }\end{array}$ & $\begin{array}{l}\text { Divagar, } \\
\text { abstraerse, } \\
\text { sonar, añorar, } \\
\text { fantasear, } \\
\text { evocar, } \\
\text { relajarse, } \\
\text { divertirse, jugar }\end{array}$ & $\begin{array}{l}\text { Privacidad, } \\
\text { intimidad, } \\
\text { espacios de } \\
\text { encuentro, } \\
\text { tiempo libre, } \\
\text { ambientes, } \\
\text { paisajes }\end{array}$ \\
\hline CREACION & $\begin{array}{l}\text { Pasión, } \\
\text { voluntad, } \\
\text { intuición, } \\
\text { imaginación, } \\
\text { audacia, } \\
\text { racionalidad, } \\
\text { autonomía, } \\
\text { inventiva, } \\
\text { curiosidad }\end{array}$ & $\begin{array}{l}\text { Habilidades, } \\
\text { destrezas, } \\
\text { método, } \\
\text { trabajo }\end{array}$ & $\begin{array}{l}\text { Trabajar, } \\
\text { inventar, } \\
\text { construir, } \\
\text { idear, } \\
\text { componer, } \\
\text { diseñar, } \\
\text { interpretar }\end{array}$ & $\begin{array}{l}\text { Ambitos de } \\
\text { producción y } \\
\text { retroalimentación, } \\
\text { talleres, } \\
\text { ateneos, } \\
\text { agrupaciones, } \\
\text { audiencia, } \\
\text { espacios de } \\
\text { expresión, } \\
\text { libertad } \\
\text { temporal } \\
\end{array}$ \\
\hline
\end{tabular}




\begin{tabular}{|c|c|c|c|c|}
\hline \begin{tabular}{|l|} 
Necesidades \\
según categorias \\
existenciales
\end{tabular} & SER & TENER & HACER & ESTAR \\
\hline \multicolumn{5}{|l|}{$\begin{array}{l}\text { Necesidades } \\
\text { según categorias } \\
\text { axiológicas }\end{array}$} \\
\hline IDENTIDAD & $\begin{array}{l}\text { Pertenencia, } \\
\text { coherencia, } \\
\text { diferencia, } \\
\text { autoestima, } \\
\text { asertividad }\end{array}$ & $\begin{array}{l}\text { Símbolos, } \\
\text { lenguaje, } \\
\text { hábitos, } \\
\text { costumbres, } \\
\text { grupos de } \\
\text { referencia, } \\
\text { sexualidad, } \\
\text { valores, } \\
\text { normas, roles, } \\
\text { memoria } \\
\text { histórica, } \\
\text { trabajo } \\
\end{array}$ & $\begin{array}{l}\text { Comprometerse, } \\
\text { integrarse, } \\
\text { confundirse, } \\
\text { definirse, } \\
\text { conocerse, } \\
\text { reconocerse, } \\
\text { actualizarse, } \\
\text { crecer }\end{array}$ & $\begin{array}{l}\text { Socio-ritmos, } \\
\text { entornos de la } \\
\text { cotidianeidad, } \\
\text { ámbitos de } \\
\text { pertenencia, } \\
\text { etapas } \\
\text { madurativas }\end{array}$ \\
\hline LIBERTAD & $\begin{array}{l}\text { Autonomía, } \\
\text { autoestima, } \\
\text { voluntad, } \\
\text { pasión, } \\
\text { asertividad, } \\
\text { apertura, } \\
\text { determinación, } \\
\text { auda cia, } \\
\text { rebeldia, } \\
\text { tolerancia } \\
\end{array}$ & $\begin{array}{l}\text { Igualdad de } \\
\text { derechos }\end{array}$ & $\begin{array}{l}\text { Discrepar, } \\
\text { optar, } \\
\text { diferenciarse, } \\
\text { arriesgar, } \\
\text { conocerse, } \\
\text { asumirse, } \\
\text { desobedecer, } \\
\text { meditar }\end{array}$ & $\begin{array}{l}\text { Plasticidad } \\
\text { espacio- } \\
\text { temporal }\end{array}$ \\
\hline
\end{tabular}

posibilidades. Proponemos, a título de hipótesis, distinguir estos cinco tipos: I) violadores o destructores; II) pseudosatisfactores; III) satisfactores inhibidores; IV) satisfactores singulares; V) satisfactores sinérgicos.

Los violadores o destructores son elementos de efecto paradojal. Son aplicados con la intención de satisfacer una determinada necesidad, pero no sólo destruyen por completo la posibilidad de satisfacerla en un plazo mediato, sino que imposibilitan, por sus efectos colaterales, la satisfacción adecuada de otras necesidades. Así, el armamentismo, supuestamente destinado a satisfacer la necesidad de protección, en el fondo aniquila la subsistencia, el afecto, la participación y la libertad. Algo semejante sucede con el exilio forzado, la doctrina de la seguridad nacional, la censura, la burocracia o el autoritarismo. Estos elementos paradójicos parecen estar vinculados preferentemente con la necesidad de protección, la cual puede generar comportamientos humanos aberrantes, en la medida en que su insatisfacción va acompañada del miedo. El atributo que caracteriza a los violadores es que siempre son impuestos. 
Los pseudo-satisfactores son elementos que estimulan una falsa sensación de satisfacción de una necesidad determinada. Sin la agresividad de los violadores o destructores, pueden en ocasiones aniquilar, en un plazo mediato, la posibilidad de satisfacer la necesidad a que originalmente apuntan. Su atributo especial es que generalmente son inducidos mediante la propaganda, la publicidad y otros medios de persuasión.

Los satisfactores inhibidores son aquellos que por el modo en que satisfacen (generalmente sobresatisfacen) una necesidad determinada dificultan seriamente la posibilidad de satisfacer otras necesidades. Su atributo es que salvo excepciones, se hallan ritualizados en el sentido de que suelen emanar de hábitos arraigados.

Los satisfactores singulares son aquellos que apuntan a la satisfacción de una sola necesidad, siendo neutros con respecto a la satisfacción de otras necesidades. Son característicos de los planes y programas de desarrollo, cooperación y asistencia. Su principal atributo es el de ser institucionalizados, ya que tanto en la organización del Estado como en la organización civil, su generación suele estar vinculada a instituciones, sean estas Ministerios, otrás reparticiones públicas o empresas de diverso tipo.

Los satisfactores sinérgicos son los que al satisfacer una necesidad determinada estimulan y contribuyen a la satisfacción simultánea de otras necesidades. Su principal atributo es el de ser contrahegemónicos, en el sentido de que revierten racionalidades dominantes tales como la competencia y la coacción. ${ }^{30}$

\subsection{El desarrollo territorial}

Según Göske (1995): "La creciente importancia del tema del desarrollo territorial proviene de la crisis experimentada por el viejo paradigma del desarrollo inducido y de las políticas sectoriales de corte centralista, debido a las profundas transformaciones tecnológicas y a los procesos de apertura comercial que han experimentado prácticamente todas las economías nacionales, así como a la realidad de los tejidos productivos en América Latina, los cuales suelen estar constituidos en su mayoría por micro y pequeñas empresas que, muchas veces, no se encuentran incorporadas en las estructuras formales de mercado." 31 
El desarrollo de aquellos sectores económicos insertos en el núcleo globalizado de la economía por lo general no han generado efectos de difusión hacia aquellos sectores desvinculados de los mercados internacionales, dado que la lógica de funcionamiento de las empresas insertas en el núcleo globalizado de la economía es radicalmente diferente a la de aquellas empresas que se desenvuelven en los mercados internos. Sin embargo, este último tipo de empresa representa a su vez la inmensa mayoría del universo empresarial en América Latina en general y en El Salvador en particular, y tiene una alta incidencia en el empleo. Es muy común que el tejido productivo conformado por estas empresas esté relativamente desarticulado. Sin disponer de una base productiva eficientemente articulada, las oportunidades de desarrollo de las zonas en que se encuentran esas empresas son altamente limitadas. ${ }^{32}$

Entonces, pese a altas tasas de crecimiento dentro de un contexto de estabilidad macroeconómica, persisten fuertes desequilibrios, tanto en términos sociales como con respecto al rezago de regiones enteras. El crecimiento, el aumento de las inversiones e, incluso, los avances en la superación de la pobreza, suelen concentrarse en los grandes centros urbanos.

Este en efecto es el caso de El Salvador. Según el Informe sobre Desarrollo Humano para El Salvador (PNUD, 2003), El Salvador está dividido en dos países: uno urbano, relativamente moderno, que se desarrolla de manera poco ordenada, pero que ofrece mayores oportunidades económicas y sociales a la población, debido a que produce el $80 \%$ de los ingresos netos empresariales y fiscales; y otro rural, sumido en el atraso y la marginación, dependiente de la exportación de jóvenes y de las remesas que devuelven, y demandante de recursos fiscales. Según los datos de dicho Informe, las brechas sociales urbano-rurales continúan siendo gigantescas y algunas incluso han aumentado. En 1992 la pobreza total y la pobreza urbana eran 11.3 y 11.5 puntos porcentuales más altas en el área rural que en el área urbana; para 2002 tales brechas habían aumentado a 21.8 y 16.9 puntos porcentuales, respectivamente. Por otra parte, en comparación con una persona que vive en el área urbana, un habitante rural tiene en promedio una esperanza de vida casi seis años menor, la mitad de escolaridad, y la tercera parte de los ingresos. La brecha urbano-rural en cuanto desnutrición infantil ha crecido desde $8.0 \%$ en 1993 a $14.6 \%$ 
en 2003, debido a que casi todo el progreso estuvo en zonas urbanas, y la mitad de los municipios muestran desnutrición crónica en más de $30 \%$ de los escolares de primer grado.

Como una manera de responder a estas inquietudes, el desarrollo territorial se entiende como los procesos de transformación productiva y organizativa en cuyo marco el conjunto de actores sociales presentes en un determinado territorio mancomunadamente aprovechan potencialidades endógenas. Dado que las actividades empresariales no se desenvuelven en espacios neutrales, sino que están insertas en un contexto muy particular, en buena medida el potencial de desarrollo de las empresas depende de las características del territorio, que puede ser percibido como el entorno que da sustento a las actividades empresariales. ${ }^{33}$

Este entorno es construido por los mismos actores sociales y, por tanto, comprende mucho más que la existencia o no de, por ejemplo, recursos naturales. De extrema relevancia es la existencia de servicios avanzados a la producción como son, por ejemplo, institutos tecnológicos o de diseño, fuentes formales de financiamiento para las empresas locales independientemente de su tamaño, disponibilidad de mano de obra calificada, centros de capacitación laboral, etc. ${ }^{34}$

$\mathrm{Al}$ existir estos servicios al alcance del tejido productivo local, los costos de transacción que los empresarios tienen que asumir para acceder a ellos se reducen notablemente y, por ende, se aceleran los procesos de innovación. El concepto de los servicios avanzados a la producción implica una concepción más amplia acerca de la infraestructura. Se distingue entre infraestructuras básicas tangibles (carreteras, puentes, embalses, aeropuertos, etc.) e intangibles (acceso a la información, servicios de asesoría empresarial, etc.) La creación de las infraestructuras básicas, en el marco de un proceso de acondicionamiento del territorio, implica un costo relativamente alto. Por consiguiente, se requiere de la actuación coordinada entre los distintos niveles del aparato público, desde lo local hasta lo nacional. Claro está que el desafío de crear este tipo de entorno apunta a la introducción de innovaciones no solamente en el seno de la base productiva, sino que también en la institucionalidad pública.

Una definición operativa del desarrollo local para El Salvador 


\section{El desarrollo local}

En un primer momento, el desarrollo local surge como una reacción frente a los desequilibrios territoriales generados por el proceso de industrialización de posguerra en los países del norte (principalmente Europa), donde las regiones ricas e industrializadas conviven con regiones pobres y marginadas del proceso de inversión, industrialización y comercio global. Igualmente, los procesos de des-industrialización de extensas áreas, regiones y localidades europeas frente a la competencia global surgida de la nueva conformación de los procesos y las estructuras productivas, proveniente sobre todo de los países asiáticos, confrontan a regiones con decisiones estratégicas en cuanto a su dependencia de las inversiones y del comercio con el mercado externo frente a los factores endógenos con los que se cuenta.

En este sentido surgen los primeros movimientos y teorías del desarrollo local sobre todo en Francia, concibiéndose un desarrollo de "abajo hacia arriba" sobre la base de los recursos y las potencialidades endógenas, para superar la dependencia en el mercado global. A partir de este surgimiento de la importancia de lo local, los proyectos y movimientos de desarrollo local tienen un eco en los países del tercer mundo en los años setentas, aunque en un primer momento estos son concebidos como proyectos de la cooperación para el desarrollo, focalizados en territorios específicos. Actualmente, el concepto se ha ampliado para reivindicar asimismo la importancia de los factores endógenos en el proceso de desarrollo, enfocado en la satisfacción de las necesidades de las mayorías y el aumento de la calidad de vida humana en el ámbito local.

La realidad salvadoreña presenta una cantidad importante de esfuerzos de desarrollo impulsados tanto desde el ámbito nacional como desde lo local, en muchos casos con apoyo financiero internacional. Muchas de estas iniciativas surgen con mayor fuerza después los Acuerdos de Paz de 1992, los cuales ponen fin a una guerra civil de más de una década y evidencian la necesidad de reconstruir tanto la infraestructura como el tejido socio-institucional destruido por la guerra, pero asimismo responden a tendencias de carácter global, en dónde lo local es re-descubierto como un motor para dinamizar los cambios necesarios en un contexto de globalización. Esto es lo que Francisco Albuquerque llama "el redescubrimiento de la dimensión territorial", 
asociado al fenómeno de una respuesta que surge frente a los desafíos que plantea la globalización. ${ }^{35}$

Entre las iniciativas más recientes, en el ámbito nacional se pueden nombrar las bases y acciones territoriales del Plan de Nación de la Comisión Nacional de Desarrollo, la Estrategia Nacional de Desarrollo Local elaborada por un Grupo Consultivo donde participaron una serie de actores nacionales e internacionales ${ }^{36}$, y el Programa de Desarrollo Local (PDL) del Fondo de Inversión Social para el Desarrollo Local (FISDL), financiado por el Banco Interamericano de Desarrollo (BID); y en el ámbito local, los distintos Planes de Desarrollo Local elaborados por las alcaldías municipales tanto de forma individual como mancomunada. Cabe mencionar que la formulación de la mayoría de estos planes ha sido incentivada a través del apoyo financiero de la cooperación bilateral (EEUU, Alemania, etc.) y multilateral (PNUD, BID, etc.)

\subsection{Lo local entendido como territorio y como agentes de desarrollo}

Lo local está referido a la construcción socio-histórica de un territorio, entendido éste a partir de las siguientes características ${ }^{37}$ :

- Entorno donde se fraguan las relaciones sociales y económicas.

- Contexto donde la cultura y otros rasgos locales no transferibles se han ido sedimentado y afirmando en el tiempo.

- Marco donde los hombres y las empresas establecen relaciones.

- Entorno donde las instituciones públicas y privadas interactuan para regular la sociedad.

- Factor estratégico de oportunidades de desarrollo.

Este concepto del territorio obviamente va mucho más allá del espacio físico y por lo tanto abre una amplia gama de actuación a los actores territoriales en función del desarrollo del mismo. Y es que todas las actividades que se realizan en el mundo tienen una referencia territorial; y por lo tanto, lo local adquiere importancia estratégica actuando como centro de gestión del desarrollo, y esto se da fundamentalmente en tres ámbitos:

- El referido a la integración sociocultural, que posibilitaría hacer frente a la fragmentación existente en los territorios.

- El de la representación política y la gestión pública, en donde los 
actores principales son los gobiernos locales, por su ventaja sobre los ministerios y otros organismos centralizados del gobierno nacional, en cuanto a su mayor capacidad de representación, legitimidad y flexibilidad, lo que les puede permitir lograr los consensos necesarios para impulsar un proyecto común del territorio, así como por su adaptabilidad frente a los cambios.

- El de la productividad y competitividad económica, ya que el territorio es vital para la generación de la competitividad sistémica de las empresas. ${ }^{38}$

En este sentido, el desarrollo local requiere de una definición amplia e incluyente de todos los actores locales. Adicionalmente, las organizaciones dedicadas al desarrollo local deberían reconocer que la persona no es tan sólo el objeto del proceso de desarrollo, sino que además y fundamentalmente es el sujeto: es el individuo mismo quien debe asumir el compromiso y tener la voluntad de participar en el proceso de desarrollo local. Las organizaciones promotoras del desarrollo local por tanto se deberían reconocer como facilitadores y orientadores del proceso, pero los sujetos más importantes del proceso son los miembros de cada localidad.

Con esto en mente, se puede afirmar que en el proceso de desarrollo local sostenible intervienen una gran variedad de actores, los cuales se pueden categorizar de la siguiente manera: Organizaciones comunales, gobierno local (municipalidades), organismos de gobierno central, organizaciones privadas para el desarrollo, organismos financieros y de cooperación internacional, sector académico-científico, sector laboral y empresa privada.

\subsection{Una definición operativa del desarrollo local}

Los procesos de desarrollo local deben surgir desde las potencialidades de las comunidades locales para satisfacer sus propias necesidades humanas, a partir del conocimiento de las oportunidades y desafíos existentes para el aprovechamiento sostenible de su entorno. Esto puede lograrse al combinar los conceptos de la matriz de las necesidades humanas de Manfred Max-Neef, junto con el concepto del ordenamiento territorial, entendido como el análisis de la interacción de los sistemas socio-económicos o antrópicos y los sistemas biofísicos o naturales sobre un determinado territorio. De esta manera, la apli- 
cación en un ámbito local de la metodología participativa utilizada para la definición de la matriz de las necesidades humanas de MaxNeef, serviría para guiar al proceso de investigación interdisciplinario en torno a la capacidad actual y potencial de los sistemas para la satisfacción de las necesidades identificadas. Para ser exitoso, esto requeriría de un diálogo permanente entre los actores del desarrollo local y el equipo de investigación interdisciplinario, que permita ir construyendo un conocimiento compartido sobre alternativas de desarrollo.

En definitiva, una definición operativa del desarrollo local en El Salvador implica adoptar un enfoque de desarrollo humano en el ámbito sub-nacional, regional y local, que permita medir el impacto de los procesos a través de indicadores estandarizados y comparables con el ámbito nacional e internacional, y a la vez un enfoque territorializado que permita articular a los actores en las distintas regiones del país a través de metas y objetivos comunes. Habría que agregar que en el caso del desarrollo local, su éxito dependerá de la forma de articulación que se logre establecer entre los distintos actores al interior de un territorio para lograr definir un proyecto común, así como de la capacidad de dichos actores de generar dinámicas económicas locales que logren insertarse en el contexto de la economía global. En otras palabras, se trata de procesos de desarrollo basados en los recursos y potencialidades propias de los territorios, y capaces a la vez de articular recursos y potencialidades fuera de sus territorios.

El desarrollo local se refiere a la mejora continua de la calidad de vida humana, partiendo de los factores internos de cada localidad, y su articulación con dinámicas en el ámbito regional, nacional e internacional. No está de más decir, aunque parezca obvio, que el desarrollo local lo hace cada persona miembro de una comunidad en su localidad. El punto importante a recalcar en esta afirmación es la responsabilidad que tiene cada miembro de la comunidad en la mejora continua de la calidad de vida personal y de las condiciones de vida colectivas, y por tanto la importancia que adquiere la participación y el compromiso de cada individuo dentro de su comunidad. En términos conceptuales, el desarrollo local requiere del impulso simultáneo de una diversidad de acciones, tales como el combate a la pobreza, el desa- 
rrollo político-institucional, el ordenamiento territorial y la protección del medio ambiente, la dinámica empresarial y la producción. Este impulso se debe dar de una forma integral y coordinada, con amplia participación, y partiendo del principio de que los problemas locales deben ser resueltos localmente. Además, el componente económico-productivo se reconoce como el factor estratégico para la autodeterminación y el desarrollo sostenible de la localidad.

Para lograr esto, es necesaria la conjugación permanente de tres elementos: la planificación, la participación y la ejecución de las acciones acordadas. Si el fin último del desarrollo sostenible local es la mejora de la calidad de vida humana, los resultados del proceso deben responder a las necesidades prioritarias de la población. Esto implica optimizar y racionalizar el empleo de los escasos recursos (eficiencia), el logro real de esos objetivos y la solución de las necesidades comunitarias (eficacia), la satisfacción y receptividad de los usuarios o destinatarios de los proyectos y los servicios (efectividad), el impacto de las acciones en los diferentes sectores sociales y particularmente en los objetivos de reducción o mitigación de los niveles de pobreza (equidad), y la permanencia de los beneficios en el tiempo (sostenibilidad). ${ }^{39}$

En términos de los procesos de desarrollo local, estos deberían adoptar tres grandes ejes conceptuales y metodológicos, siendo estos la participación, la educación, y la producción.

El eje de la participación se debería fundamentar en un autodiagnóstico que siente las bases del proceso de desarrollo local. Estos diagnósticos deberían tener un carácter crítico, orientado al análisis de las causas a problemas apremiantes para las comunidades, tales como la falta de agua potable, el mal acceso a servicios de salud y educación, ingresos familiares insuficientes, entre otros. A partir del análisis de causas, los problemas se priorizan, y con la ayuda de expertos en la materia, se elaboran propuestas de solución. Finalmente, se define un plan de acción, en el que los procesos son pormenorizados, identificando los resultados o metas esperadas (en términos cuantitiativos), y definiendo responsabilidades (incluyendo las instancias de seguimiento y evaluación del proceso), así como los recursos necesarios para lograrlas (financieros y humanos), tanto locales como externos. Vale la pena resaltar que el éxito de proyectos en términos de su sostenibilidad se fundamentará en el interés mostrado por las 
comunidades, la participación pública, y la apropiación del proceso por parte de los participantes en esta etapa crítica de diagnóstico y planificación. La participación también es fundamental en el seguimiento y en la contraloría social de los proyectos de desarrollo.

El eje de la educación corta transversalmente los ejes de participación y producción. Un primer principio que aplica es que la educación debe responder a las necesidades de conocimientos que se van definiendo a partir del mismo autodiagnóstico. En otras palabras, la educación se debería adecuar a las realidades, inquietudes y necesidades de los participantes. Un segundo principio es el carácter integral que debe tener el eje de educación, para lo cual debe cubrir tres áreas básicas, siendo éstas el conocimiento, las destrezas, y el saber ser. El conocimiento se refiere al aprendizaje por parte de los participantes, relacionado a los procesos que le interesan a la comunidad y a los temas que han surgido durante el diagnóstico. Por ejemplo, en una comunidad rural orientada a la producción agrícola, les pueden interesar conocimientos sobre los procesos relacionados a plantas y suelos, así como aspectos de salud humana que aquejan a la población. Las destrezas se refieren a la aplicación de los conocimientos adquiridos. Esta área aplica sobre todo en el eje de producción, ya que el énfasis es en la efectividad y eficiencia de las prácticas individuales frente a las necesidades de producción.

Por ejemplo, en la misma comunidad rural, las destrezas estarían relacionadas a prácticas para la conservación de suelos, la diversificación de las cosechas, la comercialización de las mismas, etc. Las destrezas también se aplican en la organización comunitaria, poniendo en práctica la habilidad de gestionar fondos y de ampliar sus fuentes de financiamiento. El saber ser se refiere al reconocimiento y a la práctica de valores. Aquí aplican valores conducentes al desarrollo sostenible, tales como la autodeterminación, la participación, la voluntad de aprender, el liderazgo, la solidaridad, etc.

Finalmente, el eje de la producción se debería fundamentar en la satisfacción de las necesidades humanas de las poblaciones locales, incluyendo la elevación de las condiciones socioeconómicas, a partir del fortalecimiento de las capacidades productivas y empresariales de los agentes locales. Este fortalecimiento puede consistir en la creación de agencias de desarrollo económico local, la creación de líneas

\section{3}

Una definición operativa del desarrollo local para El Salvador 
de crédito administradas por asociaciones de crédito locales, la capacitación para la diversificación agrícola y extensión de buenas prácticas, así como de la construcción de infraestructura de apoyo a la producción, entre otras.

\subsection{Integración sistémica e indicadores}

El "sistema de desarrollo local" se debe entender como la interacción entre los distintos componentes que permiten el fin último, entendido como la mejora de la calidad de vida humana en un ámbito territorial particular. En este sentido, los seres humanos se tienen que ver a partir de su relación e interacción con los sistemas que afectan su bienestar o calidad de vida.

En términos de los indicadores que se pudieran utilizar para medir el desarrollo local, estos deberían ser suficientemente amplios para incorporar las múltiples dimensiones del mismo, incorporando a la vez una serie de criterios para valorar su aproximación a los valores y principios que se consideren relevantes para la calidad de vida local.

A manera de ejemplo, se presenta la siguiente matriz de posibles indicadores de desarrollo local:

\begin{tabular}{|c|c|c|}
\hline Dimensión del desarrollo & Posibles Indicadores & Criterios \\
\hline Humana & $\begin{array}{l}\text { Matriz de necesidades } \\
\text { y satisfactores de } \\
\text { Max-Neef } \\
\text { - Índice de Desarrollo } \\
\text { Humano } \\
\text { - Indicadores de derechos } \\
\text { humanos } \\
\end{array}$ & $\begin{array}{l}\text { - Idoneidad de los } \\
\text { satisfactores utiliza- } \\
\text { dos } \\
\text { - Calidad de vida } \\
\text { - Libertad } \\
\text { - Justicia }\end{array}$ \\
\hline Socio-política & $\begin{array}{l}\text { Índices de participación } \\
\text { ciudadana } \\
\text { - Índices de rendición } \\
\text { de cuentas }\end{array}$ & $\begin{array}{l}\text { - Representatividad } \\
\text { Equidad de género } \\
\text { Transparencia }\end{array}$ \\
\hline Económica & $\begin{array}{l}\text { - Cantidad y tipo de } \\
\text { empresas } \\
\text { - Îndices de empleo } \\
\text { - Distribución del ingreso }\end{array}$ & $\begin{array}{ll}\text { - Rentabilidad } \\
\text { - } \text { Equntidad y calidad } \\
\text { Equidad }\end{array}$ \\
\hline Ambiental & $\begin{array}{l}\text { - Cantidad y calidad de } \\
\text { los recursos naturales } \\
\text { (RRNN) } \\
\text { - Uso actual y potencial } \\
\text { de los RRNN }\end{array}$ & $\begin{array}{l}\text { - Sostenibilidad } \\
\text { - Productividad }\end{array}$ \\
\hline Etc. & & \\
\hline
\end{tabular}


1. Entendida la globalización como la apertura hacia fuera, caracterizada por un mayor flujo de bienes, servicios, información e incluso personas (migración); y la localización como la apertura hacia adentro, o concertación de opciones estratégicas para determinados territorios, cada uno con actores y características particulares, que necesitan de mecanismos adecuados de inclusión y participación.

2. Rostow, W.W. How It All Began: Origins of the Modern Economy, Londres, 1975. Citado en Arndt, H.W. "La prehistoria (hasta 1945)", cap. 2, en Desarrollo económico. La bistoria de una idea, Ed. Rei, Buenos Aires, abril 1992, p. 13.

3. Harry S. Truman, Discurso de Investidura, 20 de enero de 1949, en Documents on American Foreign Relations (Documentos sobre las Relaciones Exteriores Norteamericanas), Connecticut: Princeton University Press, 1967. Citado en Esteva, G. "Desarrollo", en Sachs, W (ed.), Diccionario del desarrollo. Una guia del conocimiento como poder, Perú, PRATEC, 1996 (publicado por primera vez en 1992), http://www.ivanillich.org/Lidicc4.htm

4. Arndt, H.W. "La prehistoria (hasta 1945)", cap. 2, en Desarrollo económico. La bistoria de una idea, Ed. Rei, Buenos Aires, abril 1992, pp. 9-51.

5. Ob. Cit.

6. Rubio Fabian, R. "Comentarios críticos a las teorías del desarrollo predominantes. Hacia otro concepto del desarrollo de las sociedades." Realidad, Noviembre - diciembre 1991

7. Blomstrom, M. y Hettne, B. "Surgimiento de la teoría moderna del desarrollo", cap. 1, en "La teoría del desarrollo en transición," FCE, México, 1990, pp. 1740

8. Myrdal, Gunnar. Economic Theory and Under-Developed Regions. Duckworth, Londres, 1957. p. 80

9. Rostow, W.W. The stages of economic growth, Cambridge University Press, 1960.

10. Rubio Fabian, R. "Comentarios críticos a las teorías del desarrollo predominantes. Hacia otro concepto del desarrollo de las sociedades." Realidad, Noviembre - diciembre 1991.

11. Esteva, G. "Desarrollo", en Sachs, W (ed.), Diccionario del desarrollo. Una guia del conocimiento como poder, Perú, PRATEC, 1996 (publicado por primera vez en 1992), http://www.ivanillich.org/Lidicc4.htm.

12. Naciones Unidas, 'Report of the 1969 Meeting of Experts on Social Policy and Planning' (Informe del Encuentro de Expertos en Política Social y Planificación 1969), en International Social Development Review, No. 3, 1971. Ob. Cit.

13. Karl Polanyi, The Great Transformation, Nueva York: Rinehart and Co., 1944. (En español, La gran transformación, México: Fondo de Cultura Económica, 1990).

14. Arndt, H.W. "La prehistoria (hasta 1945)", cap. 2, en Desarrollo económico. La bistoria de una idea, Ed. Rei, Buenos Aires, abril 1992, pp. 9-51.

15. List, F. Sistema Nacional de Economía Política. Aguilar, Madrid, 1955.

16. Karl Polanyi, 'On belief in economic determinism' (Sobre la fe en el determinismo económico), Sociological Review, Vol . XXXIX, sección primera,

Una definición operativa del desarrollo local para El Salvador 
1947. Citado en Esteva, G. "Desarrollo", en Sachs, W (ed.), Diccionario del desarrollo. Una guia del conocimiento como poder, Perú, PRATEC, 1996 (publicado por primera vez en 1992), http://www.ivanillich.org/Lidicc4.htm.

17. Ob. Cit.

18. Karl Polanyi, The Great Transformation, Nueva York: Rinehart and Co., 1944. p. 73.

19. Ob. Cit.

20. Robert S. McNamara, 'The True Dimension of the Task' (La Verdadera Dimensión de la Tarea),en International Development Review, 1970,Vol. 1. Citado en Esteva, G. "Desarrollo", en Sachs, W (ed.), Diccionario del desarrollo. Una guía del conocimiento como poder, Perú, PRATEC, 1996 (publicado por primera vez en 1992), http://www.ivanillich.org/Lidicc4.htm.

21. La Declaración de Cocoyoc fue adoptada por los participantes en en el Simposio sobre Patrón de uso de recursos, ambiente y desarrollo, del Pnud y la Unctad, en Cocoyoc, México, en octubre de 1974. Ob. Cit.

22. Fundación Dag Hammarskjold, 'What Now? Another Development' ( ¿,Y Ahora qué ? Otro Desarrollo), numero especial de Development Dialogue, Uppsala: la Fundación,1975. (Hay edición en español). Ob. Cit.

23. Según Esteva, sin embargo, apenas se tomó en cuenta el hecho de que "esta sensata consideración lleva a un callejón sin salida en la teoría y la practica mismas del desarrollo; contiene una contradicción en los términos. Si el impulso es verdaderamente endógeno, es decir, si las iniciativas realmente provienen de las diversas culturas y de sus diferentes sistemas de valores, nada permite creer que de ellas surgirá necesariamente el desarrollo -independientemente de cómo se le defina- o incluso un impulso que lleve en esa dirección. Si se le aplica adecuadamente, la concepción lleva a la disolución de la noción misma de desarrollo, tras darse cuenta de la imposibilidad de imponer un solo modelo cultural en todo el mundo -como una conferencia de expertos de Unesco reconoció apropiadamente en 1978." Ob. Cit.

24. Informe sobre Desarrollo Humano El Salvador 2003. Programa de las Naciones Unidas para el Desarrollo (PNUD), San Salvador, 2003.

25. Ob. Cit

26. Ob. Cit.

27. Ob. Cit.

28. Esteva, G. "Desarrollo", en Sachs, W (ed.), Diccionario del desarrollo. Una guía del conocimiento como poder, Perú, PRATEC, 1996 (publicado por primera vez en 1992), http://www.ivanillich.org/Lidicc4.htm.

29. Max-Neef, M. en colaboración con Antonio Elizalde y Martín Hopenhayn. Desarrollo a Escala Humana. SERJUS, Guatemala, 1995.

30. Max-Neef, M. en colaboración con Antonio Elizalde y Martín Hopenhayn. Desarrollo a Escala Humana. SERJUS, Guatemala, 1995.

31. Göske, Joachim. "Desarrollo Territorial: Hacia un Enfoque Sistémico e Integrador", La dimensión local del desarrollo: enfoque territorial, tejido productivo local, concertación de actores y aprendizaje para la acción. Fundación Friedrich Ebert, Caupolicán Servicios Gráficos, Chile, 1995.

32. Albuquerque, F. "Competitividad Internacional - Estrategia Empresarial y Papel de las Regiones”, Serie Ensayos del ILPES, Santiago de Chile 1995, pag. 2. 
Citado en Göske, Joachim. "Desarrollo Territorial: Hacia un Enfoque Sistémico e Integrador", La dimensión local del desarrollo: enfoque territorial, tejido productivo local, concertación de actores y aprendizaje para la acción. Fundación Friedrich Ebert, Caupolicán Servicios Gráficos, Chile, 1995.

33. Albuquerque, F. "Cambio Estructural, Globalización y Desarrollo Económico", en: Desarrollo y Gestión Local, Fundación Friedrich Ebert, Santiago de Chile 1997, pag. 14. Ob. Cit.

34. Albuquerque, F. "El Proceso de Construcción Social del Territorio para el Desarrollo Económico Local”, ILPES, Santiago de Chile, pag. 4. Ob. Cit.

35. Alburquerque Llorens, Francisco. Desarrollo económico local en Europa y América Latina. Consejo Superior de Investigaciones Científicas, Madrid, 1999.

36. En la elaboración de la ENDL participaron el Fondo de Inversión Social para el Desarrollo Local, FISDL y el Instituto Salvadoreño de Desarrollo Municipal, ISDEM, agencias de cooperación bilateral como la Agencia para el Desarrollo Internacional de EEUU (USAID) y la Agencia de Cooperación Técnica Alemana (GTZ), organizaciones no gubernamentales incluyendo a la Red de ONG's para el Desarrollo Local - RDL, e instituciones multilaterales, incluyendo al Banco Interamericano de Desarrollo - BID y al Programa de las Naciones Unidas para el Desarrollo (PNUD).

37. OIT, El desarrollo local como motor de cambio. Programa DelNet, Curso de formación a distancia, Unidad Didáctica 1. Turín, Italia

38. Borja Jordi y Castells Manuel (1997) Local y global. La gestión de las ciudades en la era de la información. Santillana S.A. Taurus, Madrid.

39. PROMUDE/GTZ, Guia para la Planificación Participativa Municipal, San Salvador, 1998. 